\title{
Eden growth model for aggregation of charged particles
}

\author{
Y.V. Ivanenko ${ }^{1}$, N.I. Lebovka ${ }^{1,2 *}$, N.V. Vygornitskii ${ }^{1}$ \\ ${ }^{1}$ Institute of Biocolloidal Chemistry, NAS of Ukraine, 42, blvr. \\ Vernadskogo, Kyiv, 252142, Ukraine \\ ${ }^{2}$ Kyiv Mogyla Academy University, 2, vul. Scovorody, Kyiv, 252145, Ukraine \\ Received September 27, 2018
}

\begin{abstract}
The stochastic Eden model of charged particles aggregation in two-dimensional systems is presented. This model is governed by two parameters: screening length of electrostatic interaction, $\lambda$, and short range attraction energy, $E$. Different patterns of finite and infinite aggregates are observed. They are of following types of morphologies: linear or linear with bending, warm-like, DBM (dense-branching morphology), DBM with nucleus, and compact Eden-like. The transition between the different modes of growth is studied and phase diagram of the growth structures is obtained in $\lambda, E$ co-ordinates. The detailed aggregate structure analysis, including analysis of their fractal properties, is presented. The scheme of the internal inhomogeneous structure of aggregates is proposed.
\end{abstract}

PACS: 02.70.Lq, 68.70.+w, 75.40M

\section{INTRODUCTION}

There are a lot of computer models aimed at simulation of the natural patterns occurring in colloidal aggregation, electrochemical deposition, dielectric breakdown, bacterial colony growth, viscous fingering, spreading, etc. [1]. Such models allow to explore the process of pattern formation in real physical systems and they are based mostly on the Eden model [2] and the model of diffusion limited aggregation (DLA) [3], with due account of particles drift, convection flow, diffusion, external field influence and specific interactions between the particles. The random successive growth model [4], two-scale drift-diffusion model [5], diffusion-migration [6] and convection-migration models [7] are good examples of such models. These models describe the most important pattern morphologies observed in various nonequilibrium systems, such as DLA-like, dendritic, needle, treelike, dense-branching, compact, stingy, spiral and chiral structures [8].

The relationship between the structure and the growing pattern morphologies, growth mechanisms and peculiarities of interaction between the elements of growing structure seems to be the main problem in this field. The local interaction is introduced into the screened growth model [9], in which the growth probability is determined by multiplicative screening contributions of the occupied sites nearest to the site of growth. In this model, the fractal dimensionality of the cluster $D_{f}$ is determined by the form of the screening function and may be varied in the wide range. Ausloos et al. have studied the magnetic Eden model [10] and magnetic diffusion-limited aggregation model 111 for the Izing-like nearest-neighbour interaction between the spins. Each of these models leads to the formation of different types of aggregates for different values of parameters controlling the growth processes. However, inclusion of the long-range particle interactions, for example, of dipolar type, requires considerable CPU-resources. At present, such simulations are done only for the systems of moderate size [12]. However, this question is very important for the interpretation of the experimental data on magnetic particles aggregation [13].

The study of the models of charged particles aggregation is also of a great interest. It is especially true in relation with the study of particle surface charge effects on the mechanism of colloidal aggregation [14, space charge effects in electrochemical deposition experiments [15], pattern formation mechanism in discharge systems [16] and possible fractal structure of ball lightning [17. The presence of the long-range interactions can produce the qualitatively new effects, not usual for the systems with the short-range interactions. Thus, systems with the spatial charge distribution are characterised by the limited stability. It is well known, that the electrostatic interactions cause the loss of stability of charged droplet [18, and may cause instabilities of charged polymer systems [19], aerosols [20], etc. For the nonequilibrium growth condition, one may expect the occurrence of such phenomena as large-scale fluctuations, lacunarity or growth of finite size aggregates.

*lebovka@roller.ukma.kiev.ua 
In this work, we propose the stochastic Eden-like lattice model of charged particles aggregation. Particles overcome the electrostatic repulsive barrier created by the aggregate and stick to it due to existence of a short-range attraction force. We observe the formation of aggregates with different morphologies, such as: linear or near-linear, linear with bending, warm-like, DBM or DBM with nucleus, and compact Eden-like.The transition between the different modes of growth is governed by the two model parameters screening length, $\lambda$, and attraction energy, $E$.

The structure of the paper is as follows. In Section II we describe the model. Section III reports the results of simulation and their discussion, including examples of the clusters, phase diagram, analysis of the radial cluster structures and their fractal properties. Section [V] contains our conclusions. Appendix A presents some simple electrostatic estimations for the model.

\section{MODEL}

The model is based on the standard two-dimensional stochastic Eden model [2]. We assume that all the particles bear the same electric charge $q$. A particle located at point $\mathrm{j}$ in the proximity of the aggregate interacts with all the particles of aggregate through the long-range Coulomb repulsion, $U>0$, and short-range 'glue' attraction, $E$. We also take into account the possibility of screening of electrostatic repulsion, and introduce the screening length $\lambda$, in order to switch off the influence of the aggregate particles located further then at the distance $\lambda$ from point $j$. The energy of electrostatic repulsion may be defined as:

$$
U(j)=\sum_{i} q^{2} a_{i j} / r_{i j}
$$

where $r_{i j}$ is a distance between the $j$-point and $i$-particle of the aggregate, aij $=1$ for $r_{i j}<\lambda$ and $a_{i j}=0$ in any other cases. The cluster growth takes place on the simple square lattice. The lattice step and the particle charge are assumed to be equal to 1 . So, in this model, the cluster growth is governed by two parameters: the screening length, $\lambda$, and the attraction energy, $E$ and we may consider the values of $\lambda, U$ and $E$ as dimensionless.

A cluster grows according to the following scheme:

a) the seed particle of the cluster is placed at the lattice point $x=y=0$;

b) the cluster perimeter site, $j$, is chosen randomly (the perimeter of the cluster is a set of unoccupied lattice sites nearest to the cluster); the value of $U$ for the $j$-site is calculated and compared with the $E$-value;

c) if $U \leq E$, then the $j$-site becomes the site of a cluster, and determination of a new cluster perimeter site takes place; otherwise, if $U>E$, the $j$-site is declared as unoccupied forever;

d) then the step b) is reiterated.

These steps run until the cluster reaches either the lattice size, or the desirable size, or until the cluster growth termination. The last case may be observed when the following condition becomes true for all the sites of cluster perimeter:

$$
U>E
$$

The lattice size varied from 400x400 to 5000x5000, the maximal number of particles in the cluster did not exceed $3 \cdot 10^{5}$. The results were averaged over the $10 \div 30$ different initialisations.

\section{RESULTS AND DISCUSSIONS}

\section{A. Phase diagram}

Fig. 1 presents examples of clusters that appear at different values of the screening length, $\lambda$, and attraction energy, $E$. All patterns were obtained on the basis of cluster images on 400x400 lattice. Small circle in the centre of each pattern denotes the seed particle. The plane of $\lambda$ and $E$ values is approximately divided into several regions that correspond to the observed cluster types. These regions are schematically presented on the phase diagram in Fig. 22. The numbers of patterns of the Fig. 1 correspond to the numbers in black squares put on the phase diagram. We discern the following regions of the phase diagram:

$\mathbf{L}_{\mathbf{f}}$ is the region of linear or near-linear finite cluster growth;

$\mathbf{L b}_{\infty}$ is the region the infinite linear clusters or linear clusters with bending;

$\mathbf{W}_{\mathbf{f}}$ is the region of finite warm-like clusters; this type of structure is represented by the patterns $1,5,9,13,17$ of Fig. 11 and similar structures are reported to occur for the random successive growth model [4]; 
$\mathbf{W}_{\infty}$ is the region of infinite warm-like clusters; this structure type examples are presented by the patterns $2,6,10$, 14 of Fig. 1;

$\mathbf{D B}_{\infty}$ is the region of lacunary clusters, clusters with developed branch structure and clusters of DBM-type [8] structures growth; in this region, clusters may possess a central dense part or a nucleus (see, patterns $3,4,7,8,11$, 12, 15, 16, 18, 19, 20 of Fig. 1);

$\mathbf{C}_{\infty}$ is the region of compact clusters or compact clusters with cavities growth; in the limit $E \rightarrow \infty$ these clusters are the clusters of standard Eden model.

The case of $\lambda=\infty$ (see 21-24, Fig. 1) is shown above the main diagram (Fig. 2). There are no regions of infinite cluster growth and no compact cluster growth for $\lambda=\infty$ (except for the limiting case of $E=\infty$, when the growth of Eden-like clusters takes place).

Thus, on increasing of $E$ at fixed $\lambda$ value, we observe the following sequences of regions

$$
\mathbf{L}_{\mathbf{f}} \rightarrow \mathbf{L b}_{\infty} \rightarrow \mathbf{W f} \rightarrow \mathbf{W}_{\infty} \rightarrow \mathbf{D B}_{\infty} \rightarrow \mathbf{C}_{\infty}
$$

when $\lambda$ is finite, and

$$
\mathbf{L}_{\mathbf{f}} \rightarrow \mathbf{L b}_{\mathbf{f}} \rightarrow \mathbf{W}_{\mathbf{f}} \rightarrow \mathbf{D B}_{\mathbf{f}}
$$

when $\lambda=\infty$.

The main distinctive feature of the phase diagram in Fig. 2 is the existence of regions with finite and infinite cluster growth. It appears merely due to the long-range character of Coulomb interactions. When a cluster grows, its total charge increases and it results in increase of the electrostatic repulsion energy at the points of new particle attachment, which are located on the cluster perimeter. In the certain cases, when the cluster sufficiently large grows, the condition of cluster growth termination (2) realises for all the points of cluster perimeter. We characterise the maximal size of a cluster by its maximal radius $R_{\max }$, which is simply the distance from the central seed particle to the most remote particle of the cluster. The full manifestation of the long-range character of Coulomb interactions occurs only in the cases when the value of $\lambda$ exceeds the spatial dimension of the cluster, i.e. when $\lambda \geq R_{\max }$. This condition is always true when $\lambda=\infty$, which is the cause of only finite cluster growth.

How it is possible to discern the different regions of the phase diagram presented in Fig. 22? Some conclusions about localisation of different regions can be made on the base of the visual analysis of patterns similar to that presented on Fig. 1. For fixed $\lambda$, the increase of $E$ leads to the growth of different type clusters. Thus, in $\mathbf{L}_{\mathbf{f}}$ region, the length of cluster increases with $E$ and in $\mathbf{L}_{\mathbf{b}}$ region the number of cluster bendings increases and the length of the linear cluster part decreases with the increase of $E$. However, the visual analysis does not allow us to make an exact estimation of region localisations. This method is effective only for determination of the sharp boundary between the region $\mathbf{D B} \mathbf{B}_{\infty}$ and region $\mathbf{C}_{\infty}$. Note that this boundary can also be localised by means of simple electrostatic estimations from which we obtained for the transition energy $E_{c}$ between region $\mathbf{D B} \mathbf{\infty}_{\infty}$ and region $\mathbf{C}_{\infty}$ the following relation: $E_{c}=\pi \lambda$ (See Appendix, Eq. (A5)).

The boundaries of $\mathbf{L}_{\mathbf{f}}, \mathbf{L b}_{\infty}, \mathbf{W}_{\mathbf{f}}$ and $\mathbf{W}_{\infty}$ regions are not defined clearly and the transition from one phase to another is not sharp and appears to be rather smeared. Due to the finite-size effects near the boundaries of regions, we observe strong fluctuations of cluster sizes and morphologies. The width of such transition zones between the different regions may vary and is difficult to determine, however, in our simulations it does not exceed $\Delta E \approx 0.2$ for fixed $\lambda$. On moving from region $\mathbf{L}_{\mathbf{f}}$ to region $\mathbf{L}_{\infty}$ at fixed $\lambda$ value, the closer we are to region $\mathbf{L}_{\infty}$, the higher is the probability of an infinite linear cluster growth. Correspondingly, the probability of finite linear cluster growth is lower. For estimation purposes, the boundary line may be defined as a line of approximately equal probabilities of infinite and finite clusters formation. This is true also for the cases of $\mathbf{L} \mathbf{b}_{\infty}-\mathbf{W}_{\mathbf{f}}$ and $\mathbf{W}_{\mathbf{f}}-\mathbf{W}_{\infty}$ boundaries. Unfortunately, such approach is not acceptable for boundary localisation between $\mathbf{W}_{\infty}$ and $\mathbf{D B}_{\infty}$ regions.

To estimate the region boundaries more precisely, we have carried out the configuration analysis of cluster structures and studied the behaviour of the maximal cluster radius $R_{\max }$.

\section{B. Configuration analysis and maximal cluster radius}

Transitions between the different regions of phase diagram may be also analysed with the help of configuration analysis. The idea is to find some correlation between the pattern type and the probability of certain nearest neighbour configuration appearance in that pattern. Any cluster on a simple square lattice consists of the particles that have 1, 2, 3 and 4 nearest neighbours. For the purposes of configuration analysis, we have discerned four types of configuration presented in Fig. 3 .

It is easy to see that the configurations of type (1) correspond to the points of cluster growth termination, the linear clusters consist mostly of type (2) linear configurations, the clusters with bending and warm-like clusters are 
the mixtures of type (2) and type (3) configurations of particles and the particles of compact and branched clusters form mostly the configurations of type (4).

The fractions of particles in $(i)$ type configurations, $P_{i}$, were calculated as the ratio of the number of particles having one neighbour in configuration (1), two neighbours in configuration (2), two neighbours in configuration (3) and three or four neighbours in configuration (4) respectively, to the total number of cluster particles.

To localise more precisely the boundary between the above mentioned finite and infinite cluster regions, we have also studied the dependency of maximal cluster radius $R_{\max }$ versus attraction energy $E$ for fixed values of screening length $\lambda$. The results were averaged over $10 \div 30$ different initialisations for each chosen pair of values $\lambda, E$ and the maximal lattice size used this work was 5000x5000. A cluster reaching the lattice boundaries was considered as infinite. Such clusters were observed only for $\lambda<\infty$, while, as it is mentioned above, for $\lambda=\infty$ all clusters are only finite, they stop to grow far beyond the limits of our system.

Figures 4 . cluster radius, $R_{\max }$ versus $E$ (b) for $\lambda=30, \infty$, respectively. These figures correspond to the continuus move along the $E$ axis across the phase diagram presented at Fig. 2 at fixed values of $\lambda$.

Note that at finite $\lambda$ the transition between the finite and infinite growth regions in the sequence (3) is obvious at $R_{\max }$ versus $E$ curve (Fig. (1) b). This is the way to fix the transition boundaries between the $\mathbf{L}_{\mathbf{f}}-\mathbf{L b}_{\infty}, \mathbf{L b}_{\infty}-\mathbf{W}_{\mathbf{f}}$, or $\mathbf{W}_{\mathbf{f}}-\mathbf{W}_{\infty}$ regions quite reliably.

In the region of linear or near-linear finite cluster growth $\left(\mathbf{L}_{\mathbf{f}}\right)$ the considerable increase of $P_{2}$, is observed with $E$ increase with relatively weak $P_{1}, P_{3}$ and $P_{4}$, vs. $E$ dependences. Such $P_{2}$ increase is accompanied by the increase of $R_{\max }$. The $\mathbf{L}_{f}-\mathbf{L} \mathbf{b}_{\infty}$ boundaries (for $\lambda=30$,Fig. 四) or $\mathbf{L}_{\mathbf{f}}-\mathbf{L} \mathbf{b}_{\mathbf{f}}$ boundaries (for $\lambda=\infty$,Fig. 5) at $E=E_{b}(\lambda)$ approximately correspond to the region of maximal $P_{2}$ values.

We can estimate the $R_{\max }(E)$ dependence in the $\mathbf{L}_{\mathbf{f}}$ region, as well as the $E=E_{b}(\lambda)$ boundary between the $\mathbf{L}_{\mathbf{f}}$ and $\mathbf{L} \mathbf{b}_{\infty}$ regions (at finite $\lambda$ ) proceeding from the following electrostatic considerations. The mainly linear clusters grow at small values of $E$ in the $\mathbf{L}_{\mathbf{f}}$ region. In this region the particles tend to join the chain cluster at the tips where the repulsive energy $U$ is the lowest. The particles forming a linear chain of length $L$, repulse with the energy $U$ the newcomer at the edge of the chain:

$$
U \propto \ln (\lambda)
$$

when $\lambda(\leq L)$ is finite, and

$$
U \propto \ln (L)
$$

when $\lambda=\infty$.

The growth of the linear chain terminates, when the cluster growth termination condition (2) is realised, so that we have for the maximal radius at $E<E_{b}(\lambda)$ :

$$
R_{\max } \propto \exp (E)
$$

where, the $E_{b}(\lambda)$ dependency may be approximated for finite $\lambda(\lambda<200)$ by

$$
E_{b}(\lambda) \cong 1.2 \ln (\lambda) .
$$

which corresponds to the boundary line between the $\mathbf{L}_{f}$ and $\mathbf{L} \mathbf{b}_{\infty}$ regions at the diagram of Fig. 2, and $E_{b}(\lambda=$ $\infty) \cong 8.0$ is the boundary line between the $\mathbf{L}_{\mathbf{f}}$ and $\mathbf{L} \mathbf{b}_{\mathbf{f}}$ regions.

Note, that at any $\lambda$ value the Eq.(7) approximates quite well the behaviour of $R_{\max }(E)$ in the interval of $E<E_{b}(\lambda)$ (see Figs. 4b-5b).

The transition to the region $\mathbf{W}_{f}$, where the growth of finite warm-like clusters takes place (both for $\lambda=30$ and for $\lambda=\infty$ ), is accompanied by the sharp decrease of $R_{\max }$ with the simultaneous increase of the fraction of particles in type (3) configurations, $P_{3}$. We approximately localise the $\mathbf{W}_{\infty}-\mathbf{D B} \mathbf{B}_{\infty}($ for $\lambda=30)$ or $\mathbf{W}_{\mathbf{f}}-\mathbf{D B}_{\mathbf{f}} \quad($ for $\lambda=\infty)$ boundaries for the transitions between the warm-like and DBM structures at those values of $E$, which correspond to approximately the same fractions of particles in configurations of (2), (3) and (4) types $\left(P_{2} \approx P_{3} \approx P_{4} \approx 0.2 \div 0.3\right)$. Note, that in all the cases the maximum point of $P_{1}$ is localised near these values of $E$. When $E$ is sufficiently large, the type (4) configurations prevail in the transition zone between the $\mathbf{W}_{\infty}$ and $\mathbf{D} \mathbf{B}_{\infty}$ regions.

\section{Radial structure of cluster}

The mechanisms of the internal cluster structure formation, as well as the matters dealing with its morphology may be clarified by the analysis of certain cluster properties changes of in the radial direction. For this purpose, we have 
analysed the local density profiles $\rho(r)$ and the $E / U(r)$ ratio that characterise both the cluster growth stability and the possibility of cluster growth termination if the condition (2) is realised.

We define the local density profile as

$$
\rho(r)=N(r) / N_{c}(r),
$$

where $N(r)$ is the number of cluster particles inside a ring of radius $r$ and one lattice unit width, centered at a seed particle; $N_{c}(r)$ is the similar number of densely packed cluster particles. Such definition implies that the denser is the cluster, the closer is its density to 1.

Consequently, according to such definition, the value of $\rho$ corresponds to the relative density profile reduced to a density profile of densely packed cluster. This definition allowed us to suppress the long-range effects of magic numbers, that are clearly present in the density profiles of densely packed clusters on regular lattices [21].

Figures $6 / 8$ present the profiles of local density, $\rho$ (a) and $E / U$ (b) for clusters obtained at $\lambda=30,100$ and $\infty$, respectively. These dependencies were obtained at $E=10,20, \ldots, 100$ for each $\lambda$ and averaged over 10 clusters grown on 200x200 lattice (for $\lambda=30,100$ ) and 150x150 lattice (for $\lambda=\infty$ ). Thus, we have analysed the structure of clusters in the following regions: $\mathbf{W}_{\infty}, \mathbf{D B}_{\infty}$ and $\mathbf{C}_{\infty}$ (for $\left.\lambda=30\right), \mathbf{W}_{\infty}$ and $\mathbf{D} \mathbf{B}_{\infty}$ (for $\lambda=100$ ), as well as the regions $\mathbf{W}_{\mathbf{f}}$ and $\mathbf{D} \mathbf{B}_{\mathbf{f}}$ (for $\lambda=\infty$ ) (see the phase diagram in Fig. 2).

In all the cases we have observed a rather sharp and self-correlated initial decrease of $\rho$ and $E / U$ values with $r$ increase. For $\lambda=30$ and $\lambda=100$ these dependencies have minimum at $r \approx \lambda / 2$ and maximum at $r \approx \lambda$. Analysis of the $\rho(r)$ and $E / U(r)$ profiles allows us to make some general conclusions dealing with the character of internal cluster structure inhomogeneity. In the most general case, four different zones of spatial cluster structure may be discerned along the radial direction of cluster structure. (Fig.9):

zone $\mathbf{A}$ or internal compact nucleus; this nucleus zone has the density $\rho \approx 1.0$ and its radius may be estimated as $R_{n} \approx E / 4$ (See Appendix, Eq.(A4));

zone $\mathbf{B}$ or near-nucleus boundary shell; in this shell, the density drops approximately as $\rho \propto 1 / r$ or all the cases observed, and this shell is located in the region of $R_{n}<r<\lambda / 2$; the existence of this shell is connected with the growth of near-linear branches from the dense nucleus;

zone $\mathbf{C}$, or screening-induced compression shell; this shell is located in the region of $\lambda / 2<r<1.2 \lambda$ and may be present only for finite $\lambda$ 's; the density maximum here is observed at $r \approx \lambda$; this shell exists due to the cluster structure compression effects, resulting from certain screening limitation of electrostatic repulsion of particles from the most dense part of cluster(its nucleus) at distances within the above-mentioned range; correspondingly, the maximum of the $E / U(r)$ curve is observed in this zone;

zone $\mathbf{D}$, or external zone; this zone is characterised by the gradual density decrease and, in some cases, the fractal behaviour of density is observed: $\rho \propto 1 / r^{2-D_{f}}$, where $D_{f}$ is the fractal dimensionality of cluster in this zone (See Section [IID).

For the case of $\lambda=30$ (Fig. 6), the energy, $E$, increase from 10 to 100 corresponds to the gradual transition $\mathbf{W}_{\infty} \rightarrow \mathbf{D B}_{\infty} \rightarrow \mathbf{C}_{\infty}$ on the phase diagram. At $E=10$ (the lowest curve), we are at the border of $\mathbf{W}_{\infty} \rightarrow \mathbf{D B}_{\infty}$ (for example, see Fig. 1, cluster 11). The zone $\mathbf{A}$ is absent in this case, since the nucleus radius is very small $\left(R_{n} \approx E / 4=2.5\right)$. At $20<E<80$, we are in $\mathbf{D B}_{\infty}$ region (for example, see Fig. 1, cluster 12); here, each energy, $E$, increase by 10 results in a nucleus size increase, approximately, by 2.5 . In these cases, the profiles of $\rho(r)$ and $E / U(r)$ reveal somewhat nontrivial behaviour, and we can find the zones $\mathbf{A} \div \mathbf{D}$ in the cluster structure. At $E \geq 80$, we are almost near the border of $\mathbf{C}_{\infty}$ region. We observe that $\rho(r)$ and $E / U(r)$ profiles elevate with $E$ increase; these profiles decrease with $r$ without passing through maxima and minima. The dashed lines on Fig. 6 correspond to the case of $E=130$, i.e., when we are far in the $\mathbf{C}_{\infty}$ region, where the clusters are densely packed or compact. In this last case, $\rho(r) \approx 1.0$ is observed and the different zones of cluster structure may be discerned only by the character of $E / U(r)$ decrease.

The similar behaviour of $\rho(r)$ and $E / U(r)$ profiles is observed also in the case of $\lambda=100$ (Fig. 7). However, some aspects of the cluster structure are more explicit in this case. Here, we have the more prominent maxima and minima of $\rho(r)$ and $E / U(r)$ profiles, and we observe no general elevation of $E / U(r)$ curves with $E$ increase. It may be explained by the fact that such behaviour is pronounced only at sufficiently large values of $E$ in the proximity of compact growth region $\mathbf{C}_{\infty}$. We had not reached $\mathbf{C}_{\infty}$ region in our simulations: for $\lambda=100$, it starts only at $E \geq 300$.

At $\lambda=\infty$, the profiles of $\rho(r)$ and $E / U(r)$ (Fig. 8) do not pass through maxima and minima, but simply drop almost like 1/r outside the nucleus zone, A. Figures 1, 21-24 contain good examples of such clusters. The dashed lines (Fig. 9) correspond to the cases when $E=200(1)$ and 300(2). In these cases, the clusters have the large compact nuclei with the radii of $R_{n} \approx 50-75$.

We have also analysed the behaviour of the $E / U(r)$ profiles for sufficiently small values of $E$ in such regions as $\mathbf{L}_{\mathbf{f}}$, $\mathbf{L} \mathbf{b}_{\infty}$ and $\mathbf{W}_{\mathbf{f}}$ for $\lambda=30$ (Fig. 10 a) and in such regions as $\mathbf{L}_{\mathbf{f}}, \mathbf{L} \mathbf{b}_{\mathbf{f}}$ and $\mathbf{W}_{\mathbf{f}}$ for $\lambda=\infty 0$ (Fig. 10b). Each of these 
$E / U(r)$ profiles was obtained through data averaging over the 10 different clusters. In the regions of finite cluster growth, the $E / U(r)$ decreases quickly with $r$ upraise. At certain critical value of $r=R_{\max }$, we observe $E / U(r)=1$ at all the points of cluster perimeter and just at this stage the cluster stops to grow. In transition zone between the finite and infinite growth regions, $E / U(r)$ takes a certain stationary value greater than 1 .

Remarkable is the case of $\lambda=30$ and $E=4.5$ (Fig. 10a), when we get into the narrow $\mathbf{L} \mathbf{b}_{\infty}$ region on the phase diagram of Fig. 2. We see that after the fast initial decrease of $E / U(r)$, its abrupt uprise is obrerved at the certain $r \approx \lambda$, and then the $E / U(r)$ value of does not change with $r$. This condition corresponds to formation of the infinite linear clusters or linear clusters with bending.

\section{Fractal properties of clusters}

As we have shown in the previous Section III , in the general case, the clusters obtained within our model do not reveal any homogeneous properties in their radial direction. Therefore, the fractal approach is not applicable for analysis of the structure of whole cluster. Instead, we have analysed the fractal properties of cluster branches in the zone $\mathbf{D}$, or its external zone, where we observed the gradual local density decrease, which may be approximately described by the relation $\rho \propto 1 / r^{2-D_{f}}$, where $D_{f}$ is the fractal dimensionality of cluster in this local zone.

In order to estimate the $D_{f}$ value in this zone, we apply the sandbox method with shells of increasing outer radius, excluding the central cluster part of the radius $R \approx 1.2 \lambda$. We analyse the dependency of the number of particles in the shell, $N$, versus its area, $S$, using the following relation:

$$
N \propto S^{D_{f} / 2} .
$$

The results are averaged over 30 clusters for each value of $(E, \lambda)$ pair.

Figure 11 presents the results of $D_{f}$ calculations for $\lambda=10,30,50,100$ versus $E / \lambda$. On $E$ increase we observed the transition from linear $\left(D_{f}=1\right)$ to compact $\left(D_{f}=2\right)$ structures. The detailed analysis shows that this transition occurs when the system is in the region where the growth of finite $\left(\mathbf{W}_{f}\right)$ or of infinite $\left(\mathbf{W}_{\infty}\right)$ warm-like clusters takes place. Although the fractal dimensionality $D_{f}$ can be slightly lower than 2 in the $\mathbf{D B}_{\infty}$ region, however, it approaches to this value as the system approaches the $\mathbf{C}_{\infty}$ region, where the clusters are dense and their fractal dimensionality $D_{f}$ is strictly equal to 2. For the case when $\lambda=\infty$ the branches of $\mathbf{D B}_{\mathbf{f}}$ clusters do not display uniform fractal properties, but their fractal dimensionality tends to 1 when we move in radial direction from the centre. So, in this case we have $D_{f}=1$ and this behaviour is easy to understand in view of the tendency of electrostatic repulsion to linearise the branches of the cluster.

We think that the smooth character of $D_{f}$ transition on passing through the regions $\mathbf{W}_{f}$ or $\mathbf{W}_{\infty}$ results from the stochastic nature of the model we used in our simulations. Quite different behaviour of fractal dimensionality can be observed for the similar, yet deterministic in nature model of aggregation [22]. In this case we observed the sharp transition between $D_{f}=1$ for finite clusters and $D_{f}=2$ for infinite clusters.

\section{CONCLUSIONS}

We have introduced the two-parametric electrostatic Eden model that simulates the aggregation of charged particles. Cluster growth is controlled by the screening length of electrostatic interactions $\lambda$, and the energy of particle adhesion to a cluster $E$. The phase diagram in $\lambda$ versus $E$ co-ordinates reveals the regions of finite (f) and of infinite $(\infty)$ cluster growth. The model gives a variety of patterns with different morphology, including fractals. We do discern the following morphologic types: linear, linear with bending, warm-like, dense-branching and compact Eden-like. The structure compactness increases with the attraction energy $E$ increase, while the strengthening of electrostatic repulsion on $\lambda$ increase results in linear morphologies predominance. The cluster structure is not homogeneous in the radial direction and in the general case four zones with different morphological properties may be differentiated: internal compact nucleus zone, near-nucleus boundary shell, screening induced compression shell, and external shell.

\section{ACKNOWLEDGMENTS}

Authors thank Dr. N. S. Pivovarova for valuable discussions of the manuscript and help with its preparation. 


\section{APPENDIX A: SIMPLE ELECTROSTATIC ESTIMATIONS FOR THE MODEL}

We approximate a compact two-dimensional Eden cluster by uniformly charged disk of radius $R$. We calculate the energy of electrostatic interaction between the charge and compact disk-like cluster of charged particles $U$ by using numerical integration instead of summation in Eq. (1) and taking into account the homogeneous charge density distribution in the disk-like cluster with $\rho(r)=1$.

The solid line in Fig. 12 presents the dependency of the reduced electrostatic energy, $U^{*}=U / R$, against the reduced distance between the charge and the cluster centre, $r^{*}=r / R$, for $\lambda=\infty$. It is obvious that for the particle located at the cluster centre, when $r=0$, the energy is equal to $U_{o}=2 \pi R$. At large distances from the cluster centre, when $r^{*} \rightarrow \infty$, we may neglect the spatial charge distribution inside the cluster and treat the whole charged cluster as a charge $Q=\pi R^{2}$ concentrated at the centre of such cluster. In this case:

$$
U^{*}\left(r^{*} \rightarrow \infty\right)=U_{\infty}^{*}=\pi / r^{*} .
$$

The dependency of $U_{\infty}^{*}$ on $r^{*}$ is presented on Fig.12 by the dashed line. We see, that, at least, for $r^{*}>1.5$, the Eq.(A1) approximates the $U^{*}\left(r^{*}\right)$ dependency rather precisely.

If the particle is located at the circle-like cluster perimeter, where $r=R\left(r^{*}=1\right)$, the interaction energy expressions are easy to obtain in the analytical form for the arbitrary $\lambda$ value:

$$
\begin{aligned}
& U_{p}^{*}=4\left(1+\lambda^{*} \arccos \left(\lambda^{*}\right)-\sqrt{1-\lambda^{* 2}}\right) \text { for } \lambda^{*}<1 \\
& U_{p}^{*}=4 \text { for } \lambda^{*} \geq 1
\end{aligned}
$$

Here, $\lambda^{*}=\lambda /(2 R)$.

The condition $\lambda^{*} \geq 1$ from Eq. (A2b) is equivalent to the condition $\lambda \geq 2 R$. In this case, the $U_{p}$ value is defined by the interactions with all the cluster charges, without any screening limitations. Thus, for all the $R \leq \lambda / 2$, we obtain from Eq. A2b):

$$
U_{p}=4 R
$$

The stable growth of compact aggregates terminates when $E \leq U_{p}$; therefore, in the specific case of $\lambda=\infty$ we may estimate the radius of the compact nucleus as

$$
R_{n} \approx E / 4, \text { for } \lambda=\infty
$$

At $R \geq R_{n}$, the loss of compact cluster growth stability takes place and cluster ramification begins.

It is possible to estimate the $R_{n}$ values for finite screening lengths, $\lambda$ 's, by substituting $U_{p}=E$ into Eq. A2a). However, in this case the situation of infinite compact cluster growth with $R_{n}=\infty$ is possible for sufficiently large values of $E \geq E_{c}$. For the purposes of $E_{c}$ estimation, we consider Eq. A2a) when $\lambda^{*} \rightarrow 0$ (or $R \rightarrow \infty$ when $\lambda$ is a finite) and obtain $U_{p}^{*}=2 \pi \lambda^{*}$ or $U_{p}=\pi \lambda$. So, the relation for $E c(\lambda)$ may be written as follows:

$$
E_{c}(\lambda)=\pi \lambda
$$

The Eq. A5 allows us to estimate the position of the boundary between the $\mathbf{C}_{\infty}$ and $\mathbf{D B} \mathbf{B}_{\infty}$ regions on the phase diagram presented in Fig.2.

[1] F. Family, D. P. Landau (Eds.) Kinetics of Aggregation and Gelation, Noth-Holland, Amsterdam, 1984; P. Meakin in: C. Domb and J. L. Lebowitz (Eds.), Phase Transition and Critical Phenomena, Vol. 12, Academic Press, London, 1988; T. Vicsek, Fractal Growth Phenomena, World Scientific, Singapore, 1989; M. Mutsushita in: D. Avnir (Ed.), The Fractal Approach to Heterogeneous Chemistry, Wiley, Chichester, 1990.

[2] M. Eden in: F. Neyman, Proceedings of the Fourth Berkeley Symposium on Mathematics, Statistics and Probability, Vol. IV, University of California Press, Berkeley, 1961.

[3] T. A. Witten, L. M. Sander, Phys. Rev. Lett. 47 (1981) 1400.

[4] Z. Q. Wu, B. Q. Li, Phys. Rev. E, 51 (1995) R16.

[5] G. C. John, V. A. Singh, Phys. Rev. E, 53 (1996) 3920. 
[6] W. Huang, D. B. Hibbert, Phys. Rev.E, 53 (1996) 727.

[7] J. Elebacher, P. C.Searson, K. Sieradski, Phys. Rev. Lett. 71 (20) (1993) 3311.

[8] N. Nagatani, F. Sagués, J. Phys. Soc. Jpn., 59 (1990) 3447; E. Ben-Jacob, H. Shmueli, O. Shochet, A. Tenenhaum, Physica A, 187, (1992) 378; I. Mogi, S. Okubo, G. Kido, Fractals 1 (1993) 475; S. Fautrat, P. Mills, Phys. Rev.E, 53 (1996) 4990.

[9] P. A. Rikvold, Phys. Rev.A, 26 (1982) 674; P. Meakin, Phys. Rev. Lett., 51 (1983) 1119; P. Meakin, Phys. Lett.A, 107 (1985) 269.

[10] M. Ausloos, N. Vandewalle, R. Cloots, Europhys. Lett. 24 (8) (1993) 629.

[11] N. Vandewalle, M. Ausloos, Phys. Rev. E 51 (1) (1995) 597.

[12] P. M. Mors, R. Botet, R. Jullien, J. Phys. A: Math. Gen. 20 (1987) L975; R. Pastor-Satorras, J. M. Rubi, Phys. Rev. E 51 (6) (1995) 5994.

[13] G. A. Helgessen, A. T. Skjeltorp, P. M. Mors, R. Botet, R. Jullien, Phys. Rev. Lett. 61 (15) (1988) 1736; G. A. Niklasson, A. Torebring, C. Larsson, C. G. Granqvist, Phys. Rev. Lett. 60 (17) (1988) 1735

[14] A. Fernández-Barbero, M. Cabrerizo-Vilchez, R. Martinez-Garcia, R. Hidalgo-Alvarez, Phys. Rev. E, 53 (1996) 4981.

[15] J.-N. Chazalviel, Phys. Rev. A, 42 (1990) 7355.

[16] W. Breazeal, K.M. Flynn, E.G. Gwinn, Phys. Rev. E, 52 (1995) 1503.

[17] B. W. Smirnov, Phys. Rep. 152 (1987) 177.

[18] L. D. Landau, E. M. Lifshitz, Electrodynamics of continuos media, Nauka, Moscow, 1982.

[19] Y. Kantor, M. Kardar, Phys. Rev. E 51 (1995) 1299.

[20] P. C. Reist, Introduction to aerosol science, Macmillan, New York, 1984.

[21] B. W. Smirnov, Cluster Ions and van der Waals Molecules, Gordon and Breach, London, 1991.

[22] N. I. Lebovka, Ya. V. Ivanenko, N. V. Vygornitskii, Europhys. Lett. 41 (1) (1998) 19. 
FIG. 1. Examples of cluster patterns at different values of the attraction energy $E$ and screening length $\lambda$. Numbers of patterns correspond to the numbered black squares on the phase diagram on Fig. A. All patterns are displayed on the same scale $500 \times 500$.

FIG. 2. Phase diagram for the cluster growth in co-ordinates of screening length $\lambda$ versus attraction energy $E$. See the text for details.

FIG. 3. The four types of the nearest neighbour configurations of cluster growing on a square lattice discerned for the purposes of configuration analysis.

FIG. 4. The fractions of different types of particles configurations, $P_{i}, i=1 \div 4$ (a), and maximal cluster radius, $R_{\text {max }}$, (b) versus attraction energy, $E$, at $\lambda=30$. The value of $E_{b}$ determines the boundary between the $\mathbf{L}_{\mathbf{f}}$ and $\mathbf{L b}_{\infty}$ regions and may be approximated by Eq.(\$). The value of $E_{c}$ determines the boundary between the $\mathbf{D B} \mathbf{B}_{\infty}$ and $\mathbf{C}_{\infty}$ regions and may be approximated by Eq. A5.). The dashed line corresponds to the $R_{\max }(E)$ curve in the interval of $E<E_{b}$ obtained through rms

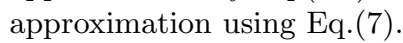

FIG. 5. The fractions of different types of particles configurations, $P_{i}, i=1 \div 4$ (a), and maximal cluster radius, $R_{\max }$,(b) versus attraction energy, $E$, at $\lambda=\infty$. The value of $E_{b}(\approx 8)$ determines the boundary between the $\mathbf{L}_{\mathbf{f}}$ and $\mathbf{L b}_{\mathbf{f}}$ regions. The dashed line corresponds to the $R_{\max }(E)$ curve in the interval of $E<E_{b}$ obtained through rms approximation using Eq. (7).

FIG. 6. The radial profiles of local cluster density, $\rho(r)$, and the energy ratios, $E / U(r)$. The results correspond to the different values of attraction energy $E=10,20,30,40,50,60,70,80,90,100$ (solid lines), $E=130$ (dashed line), the arrow shows the direction of $E$ increase. The screening length is $\lambda=30$.

FIG. 7. The radial profiles of local cluster density, $\rho(r)$, and the energy ratios, $E / U(r)$. The results correspond to the different values of attraction energy $E=10,20,30,40,50,60,70,80,90,100$ (solid lines), the arrow shows the direction of $E$ increase. The screening length is $\lambda=100$.

FIG. 8. The radial profiles of local cluster density, $\rho(r)$, and the energy ratios, $E / U(r)$. The results correspond to the different values of attraction energy $E=10,20,30,40,50,60,70,80,90,100$ (solid lines), $E=200(1), 300(2)$ (dashed lines), the arrow shows the direction of $E$ increase. The screening length is $\lambda=\infty$.

FIG. 9. The scheme of the internal structure of cluster. We display the example of cluster pattern and its local density profiles $\rho(r)$ in radial direction. The intervals of localisation of different zones $\mathbf{A}, \mathbf{B}, \mathbf{C}$ and $\mathbf{D}$ are shown, $R_{n}$ is the compact nucleus radius, $\lambda$ is the screening length.

FIG. 10. The radial profiles of the energy ratios, $E / U(r)$, for the screening length $\lambda=30(\mathrm{a}), \infty$ (b).

FIG. 11. Fractal dimension $D_{f}$ of cluster branches in the zone D (Fig.9) versus the attraction energy to screening length ratio, $E / \lambda$.

FIG. 12. The reduced electrostatic interaction energy $U^{*}=U / R$ versus the reduced distance between the centre of compact disk-like cluster and unit charge, $r^{*}=r / R$ at the screening length $\lambda=\infty$. Here $R$ is the radius of the charged disk. 


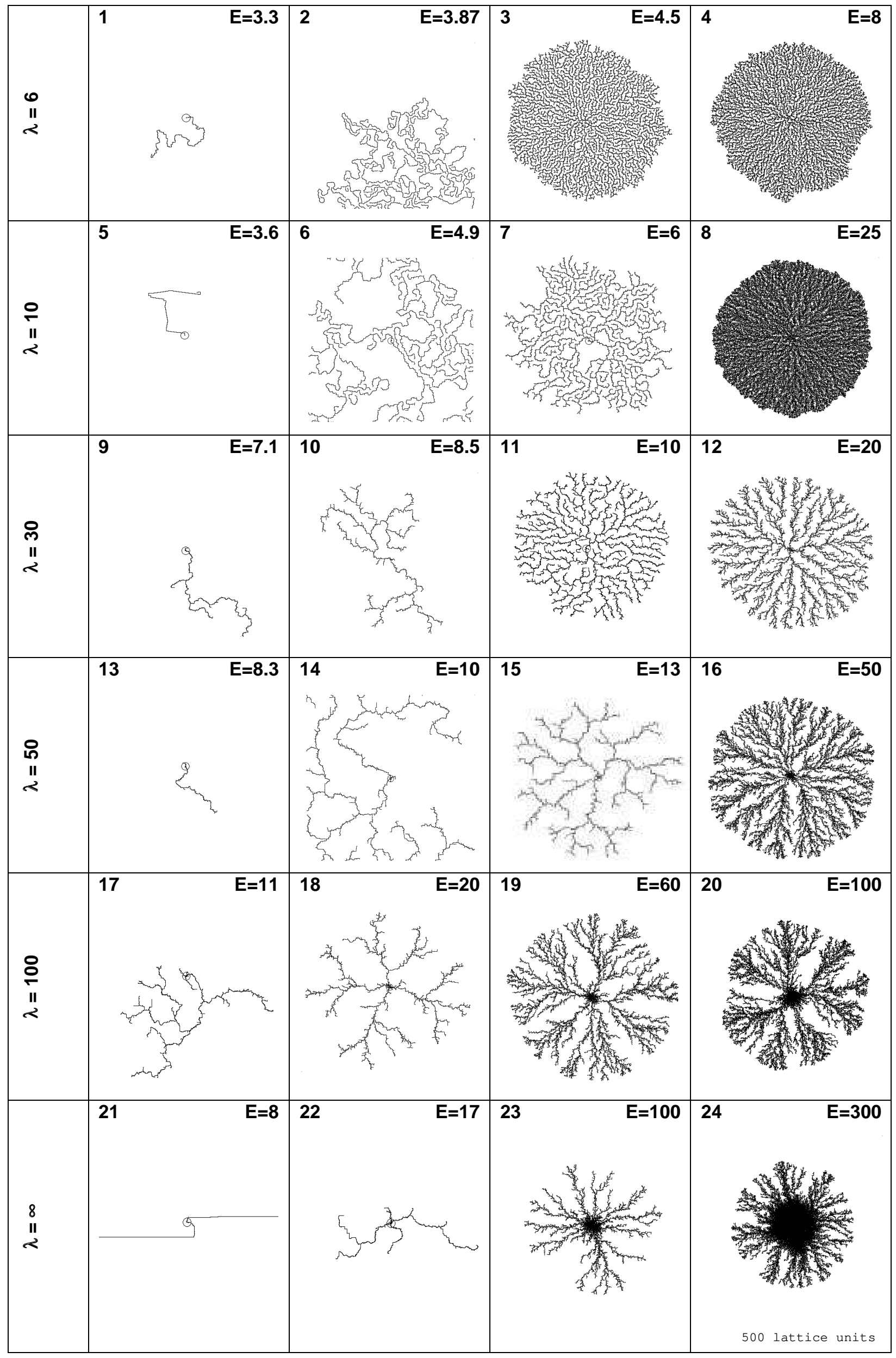




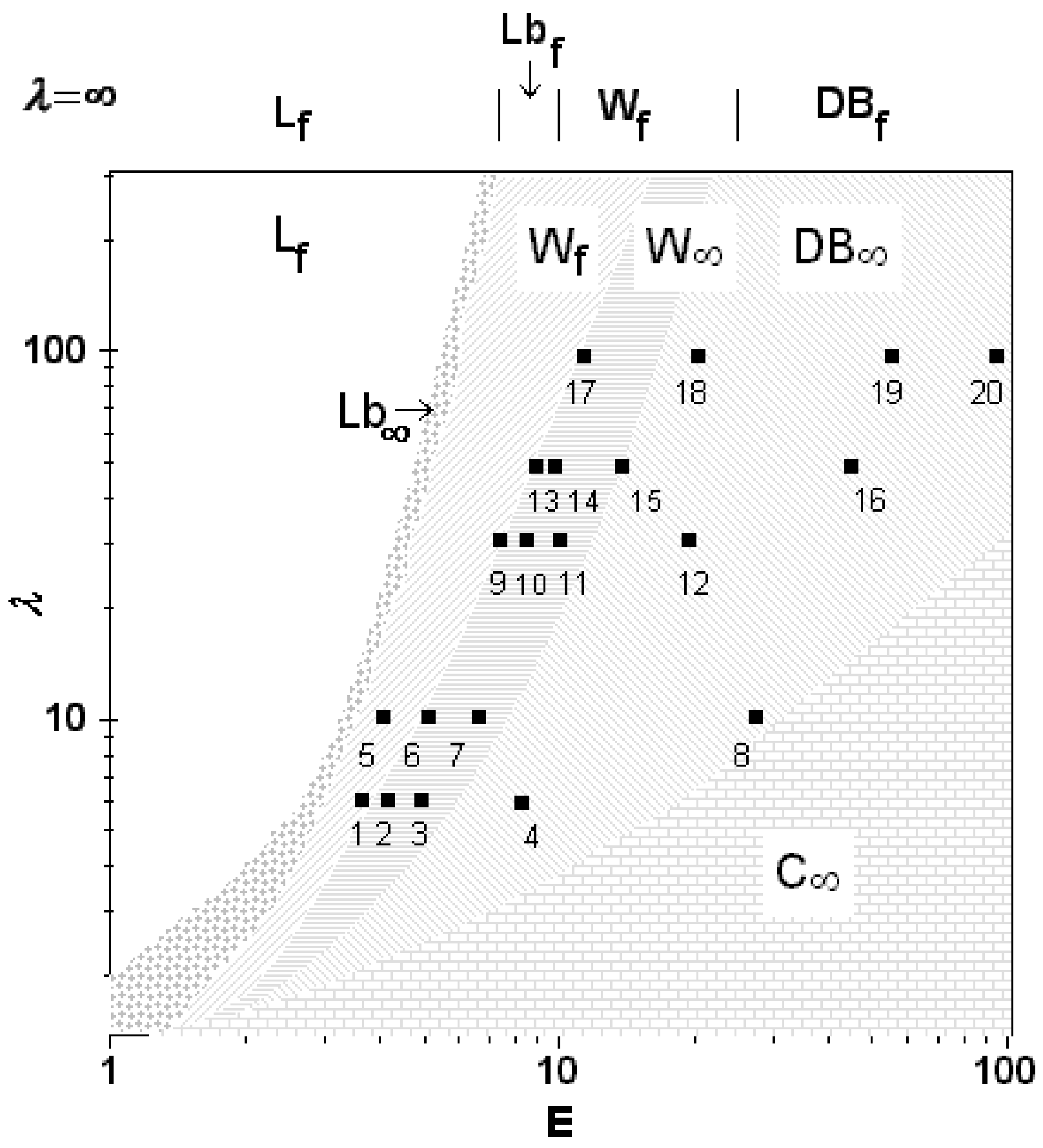


type(1)

$\infty$ type (2)

eอง type (3)

-8 type (4)

-6) 


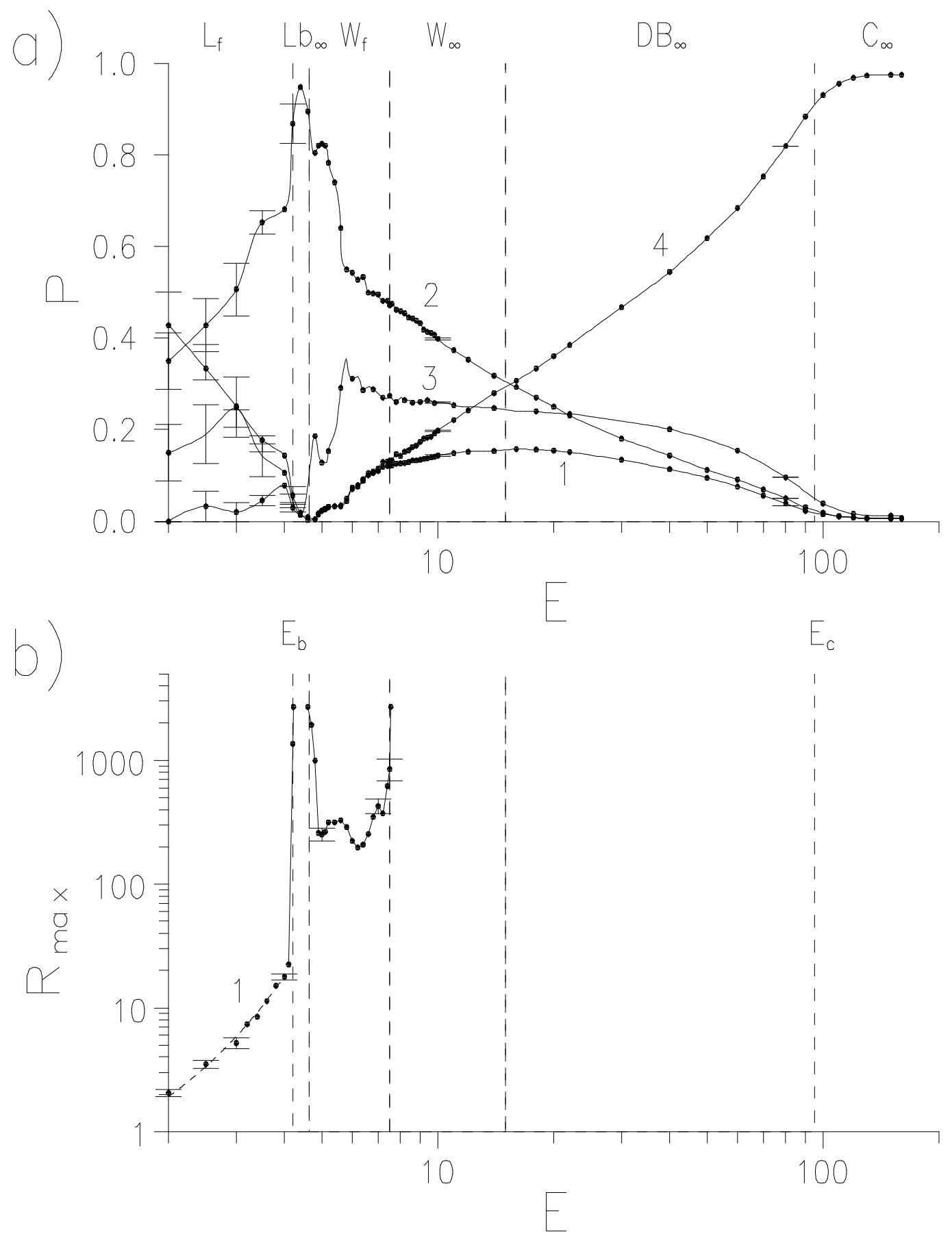




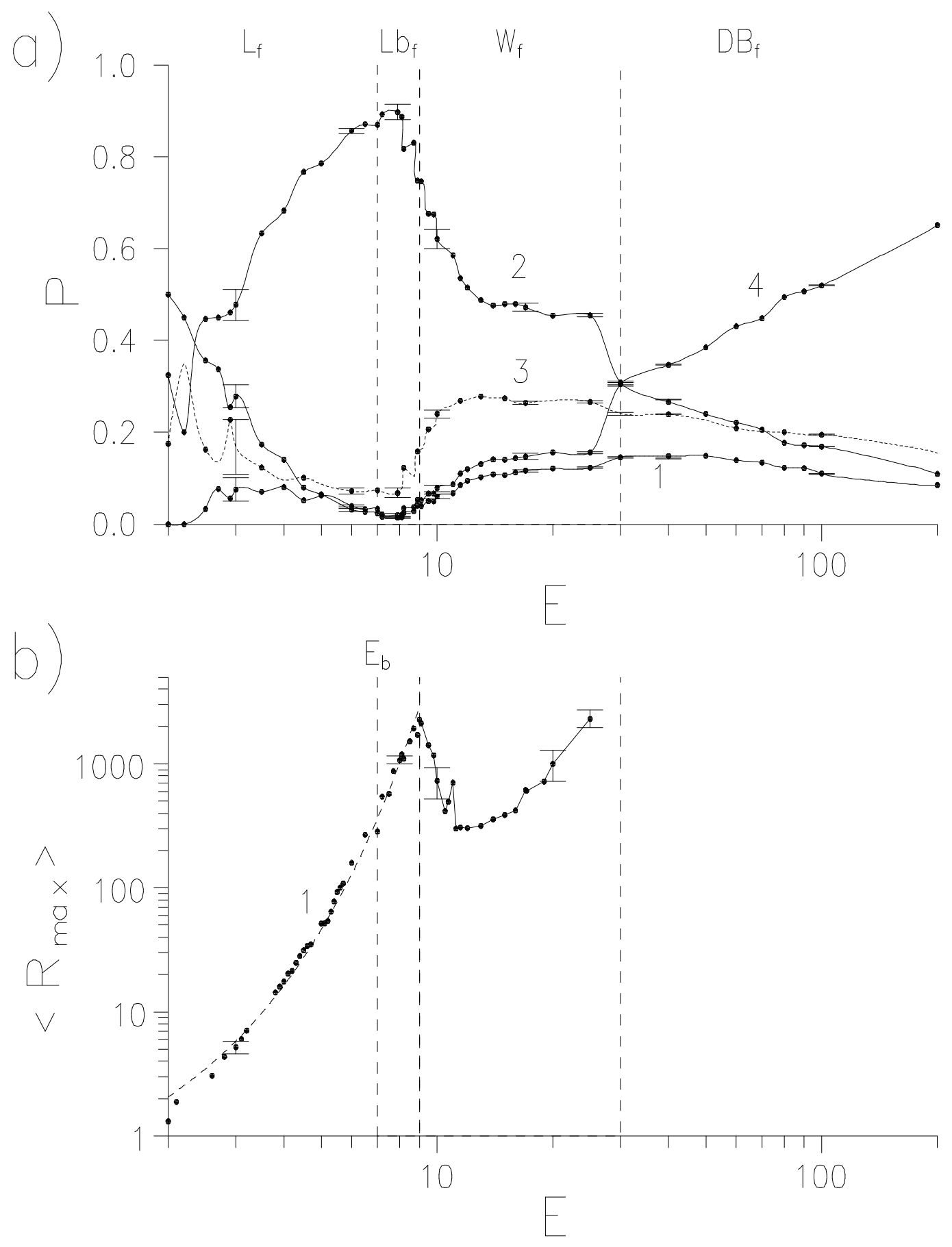



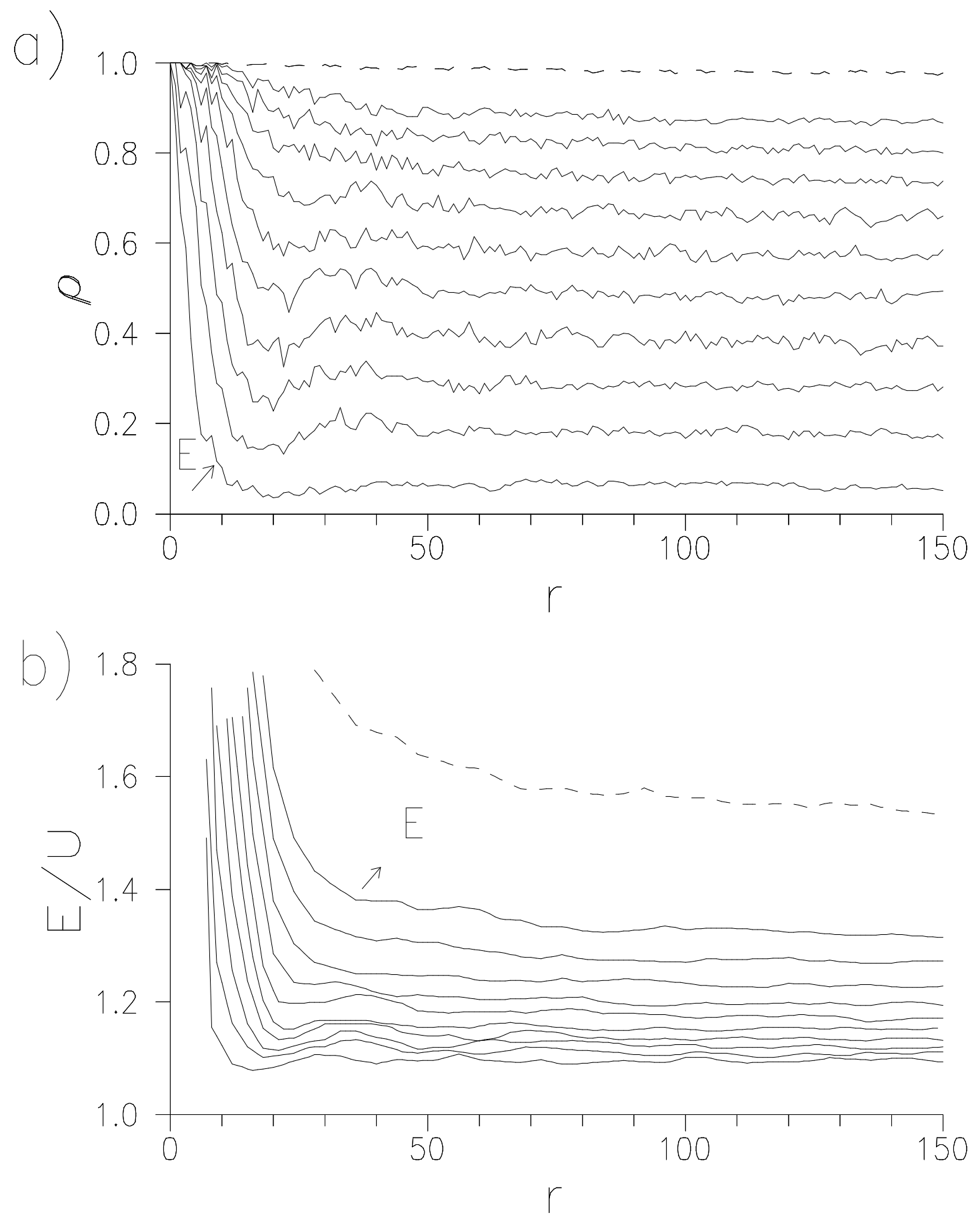


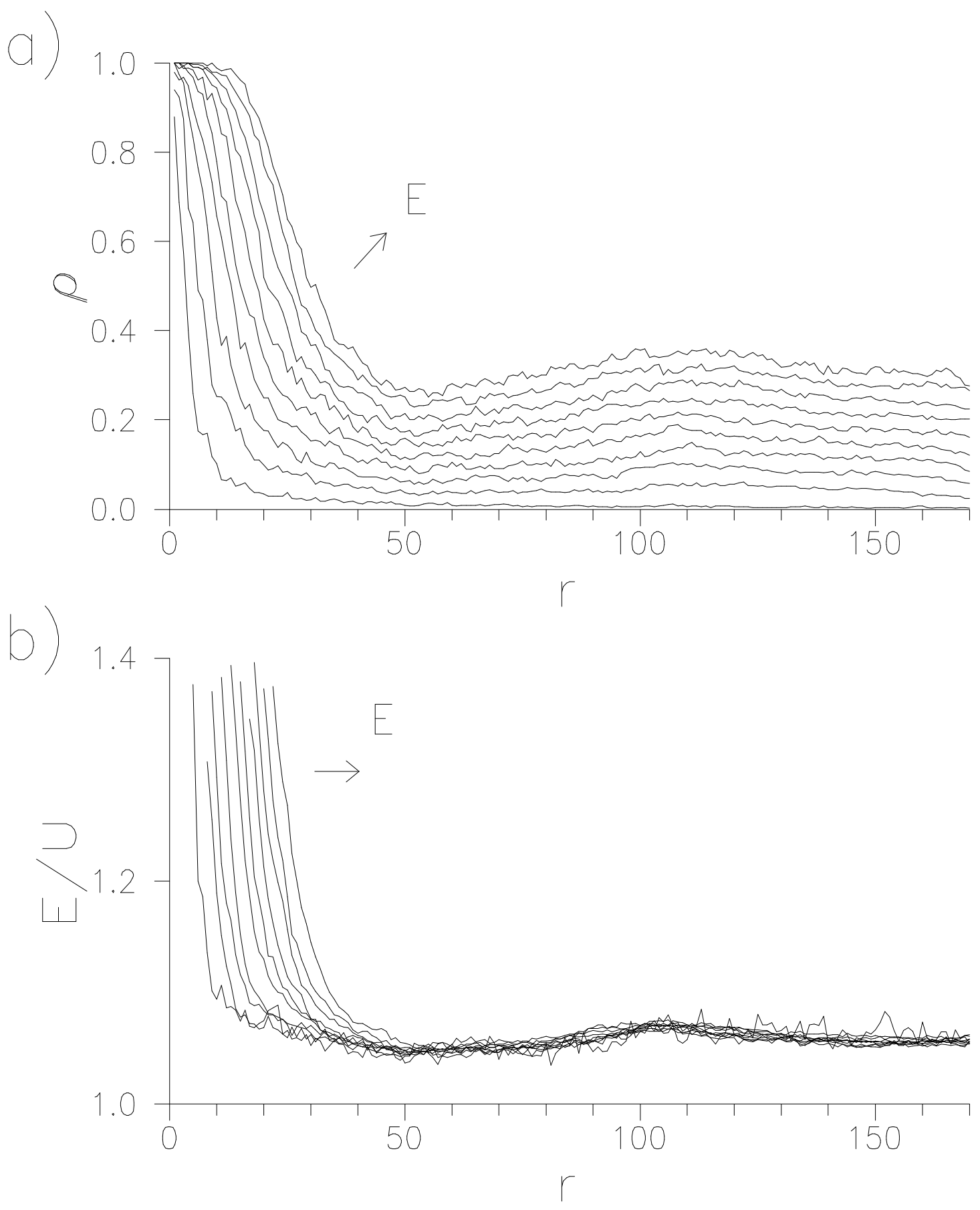




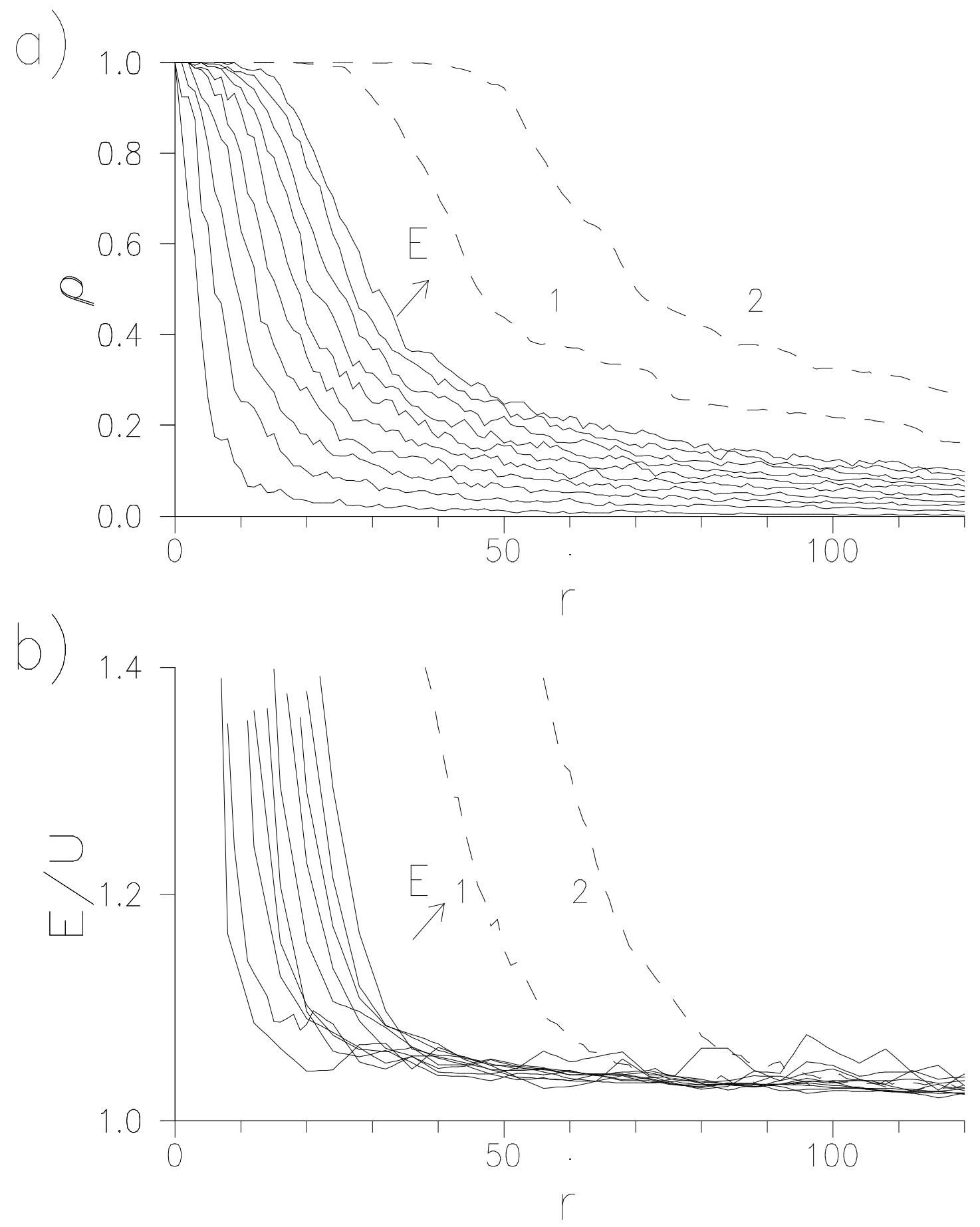




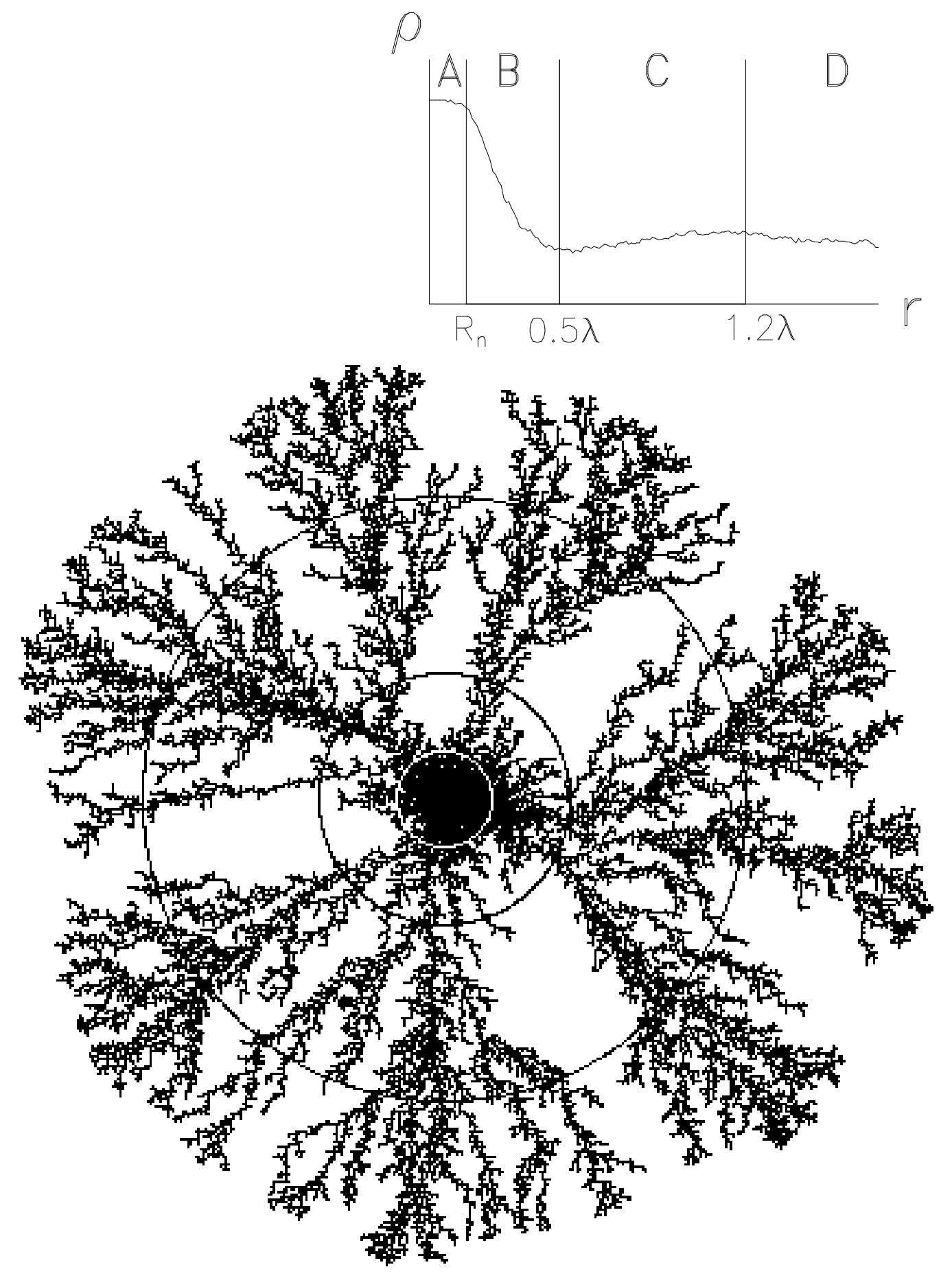




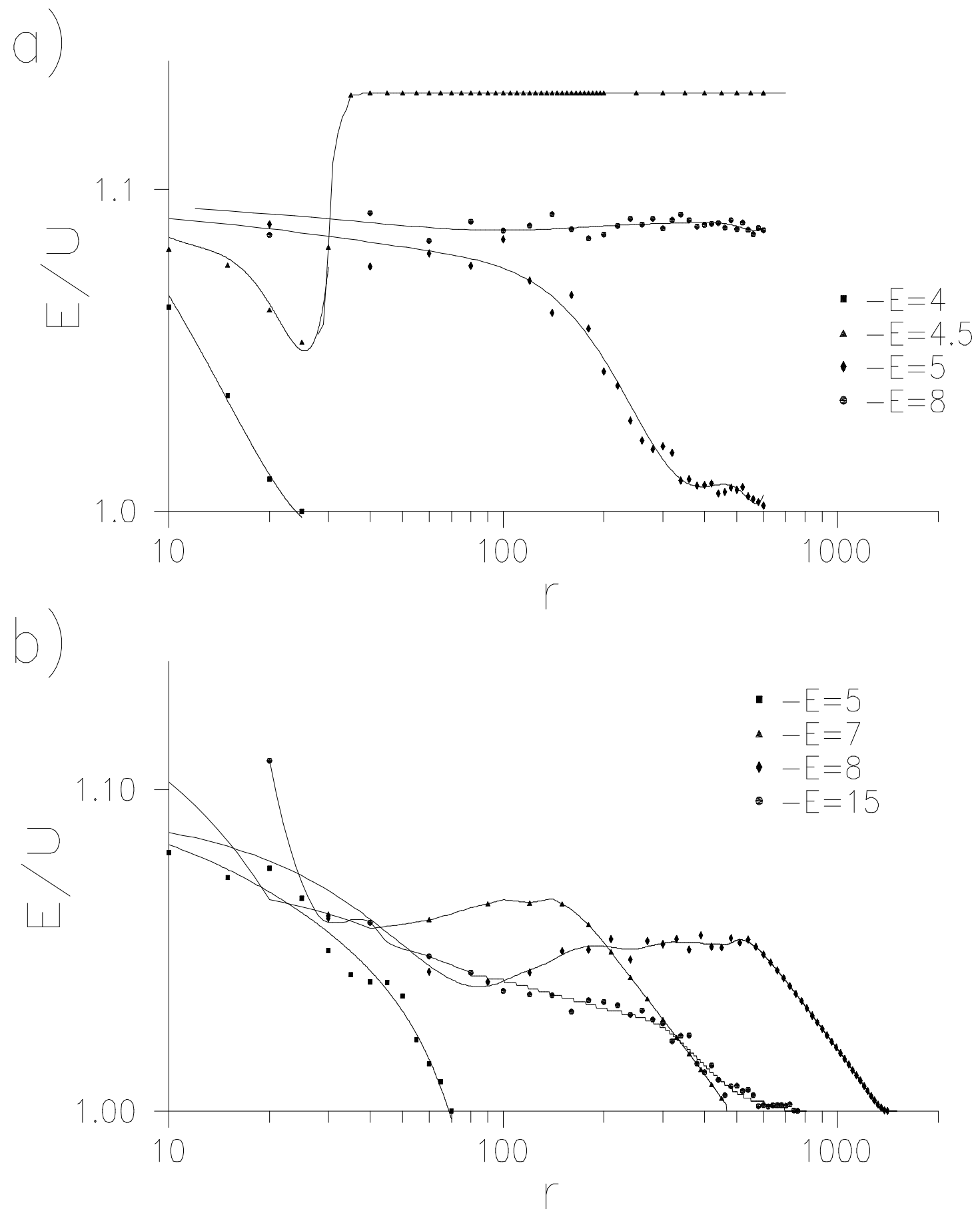




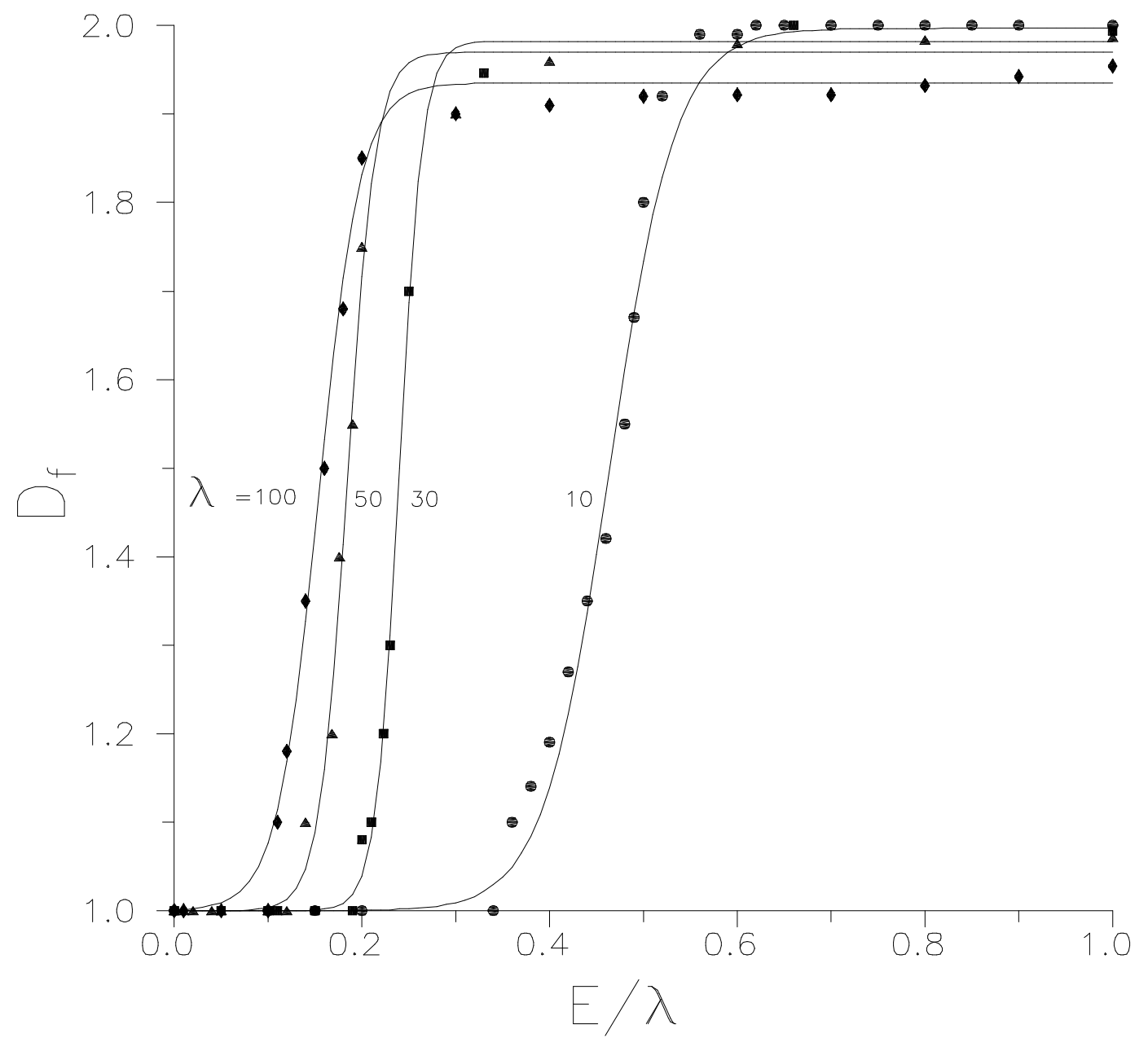




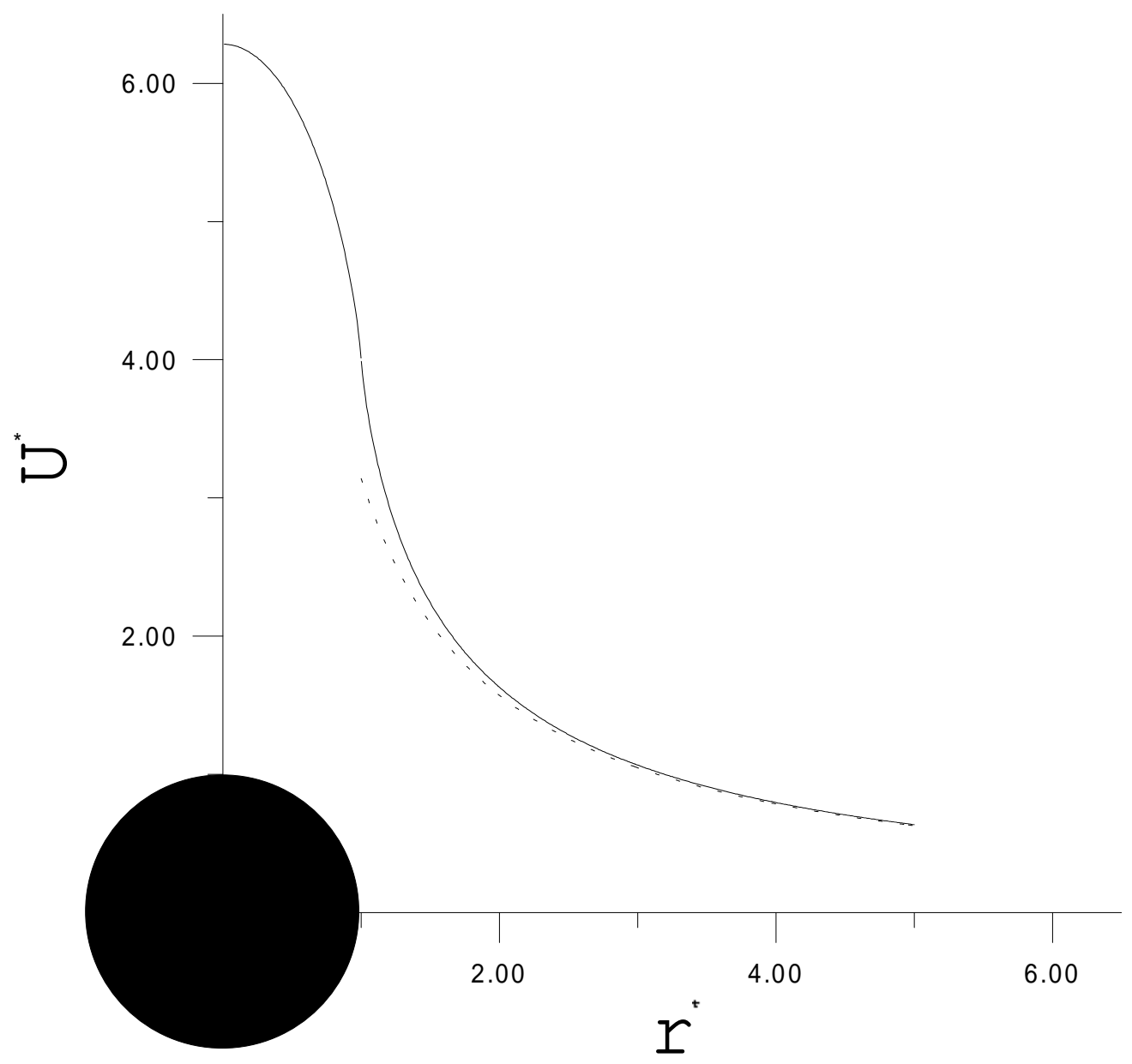

